Research Article

Special Issue: Combinatorial Matrices

Open Access

Alexander Guterman*, Elena Kreines, and Carsten Thomassen

\title{
Linear transformations of tropical matrices preserving the cyclicity index
}

https://doi.org/10.1515/spma-2020-0128

Received October 25, 2020; accepted february 9, 2021

Abstract: We combine matrix theory and graph theory methods to give a complete characterization of the surjective linear transformations of tropical matrices that preserve the cyclicity index. We show that there are non-surjective linear transformations that preserve the cyclicity index and we leave it open to characterize those.

Keywords: tropical linear algebra, cyclicity index, linear preservers

PACS: MSC[2020] 05C50; 05C38

\section{Introduction}

Let $G=(V, E)$ denote a directed graph (digraph) and let $V=V(G), E=E(G)$, and $n=|G|=|V|$ be its vertex set, its edge set, and its order, respectively. Loops are permitted in a digraph but parallel edges are not. A digraph $H$ is called a subgraph of $G$ if $V(H) \subseteq V(G)$ and $E(H) \subseteq E(G) \cap(V(H) \times V(H)$ ). A directed walk from $u$ to $v$ in $G$ is a sequence of vertices $u, w_{1}, \ldots, w_{l}, v \in V(G)$ and a sequence of edges $\left(u, w_{1}\right),\left(w_{1}, w_{2}\right), \ldots,\left(w_{l}, v\right) \in E(G)$, where vertices and edges are not necessarily distinct. A walk without repeating vertices is called simple. The length of a walk is the number of edges in it. A directed walk from $u$ to $v$ is called a closed directed walk if $u=v$. A directed cycle (or a dicycle) is a closed directed walk with no repeated vertex except for the first and last vertex. A graph is strongly connected if it has just one vertex or for any pair of vertices there is a walk from the former vertex to the latter.

Another important object of our study is a so-called max-plus semiring or a tropical semiring, which is the set $\overline{\mathbb{R}}:=\mathbb{R} \cup\{-\infty\}$ of real numbers completed by the element $-\infty$ with two binary operations $\oplus$ and $\otimes$ defined by $a \oplus b=\max \{a, b\}$ and $a \otimes b=a+b$ for all $a, b \in \overline{\mathbb{R}}$. It is easy to see that under the introduced operations $\overline{\mathbb{R}}$ is an algebraic structure, usually called a semiring. This semiring is commutative, antinegative and without zero divisors. Its neutral element with respect to the operation $\oplus$ (zero element) is $-\infty$, and the neutral element with respect to the operation $\otimes$ (unit element) is 0 . The multiplication is commutative and distributive over the addition on both sides. In addition, $-\infty \otimes a=-\infty$ for all $a \in \mathbb{R}, 0 \otimes a=a$ for all $a \in \mathbb{R}$. If for some $a, b \in \overline{\mathbb{R}}$ it holds that $a \oplus b=-\infty$, then $a=b=-\infty$. If $a \otimes b=-\infty$, then either $a=-\infty$ or $b=-\infty$. In this paper we always write + and $\cdot$ for usual operations and $\oplus$ and $\otimes$ for tropical operations.

Let $M_{n}$ denote the semiring of $n \times n$ matrices with tropical entries. Matrix addition and multiplication are defined by the usual rules, where we substitute addition and multiplication of the entries by the correspond-

\footnotetext{
*Corresponding Author: Alexander Guterman: Faculty of Mathematics and Mechanics, Moscow State University, Moscow, GSP-1, 119991, Russia; Moscow Center for Fundamental and Applied Mathematics, Moscow, GSP-1, 119991, Russia; Moscow Institute of Physics and Technology, Dolgoprudny, 141701, Russia, E-mail: guterman@list.ru

Elena Kreines: Faculty of Mathematics and Mechanics, Moscow State University, Moscow, GSP-1, 119991, Russia; Moscow Center for Fundamental and Applied Mathematics, Moscow, GSP-1, 119991, Russia, E-mail: elena.kreines@math.msu.ru Carsten Thomassen: Department of Applied Mathematics and Computer Science, Technical University of Denmark, DK-2800 Lyngby, Denmark, E-mail: ctho@dtu.dk
} 
ing tropical operations. We denote the tropical sum of matrices by $\oplus$, and the tropical product of matrices by $\otimes$, namely, for $A=\left(a_{i j}\right), B=\left(b_{i j}\right) \in M_{n}$ we define $C=\left(c_{i j}\right)=A \oplus B \in M_{n}$ and $D=\left(d_{i j}\right)=A \otimes B \in M_{n}$, where $c_{i j}=a_{i j} \oplus b_{i j}=\max \left\{a_{i j}, b_{i j}\right\}, d_{i j}=\bigoplus_{k=1}^{n}\left(a_{i k} \otimes b_{k j}\right)=\max _{k=1, \ldots, n}\left(a_{i k}+b_{k j}\right)$ for all $i, j, 1 \leq i, j \leq n$. As usual, we denote by $I_{n}$ the identity matrix, that is, it has 0 at the main diagonal and $-\infty$ elsewhere. Also, $O_{n}$ is the $n \times n$ matrix consisting only of $-\infty, J_{n}=\left(\begin{array}{ccc}0 & \ldots & 0 \\ \ldots & \ldots & \ldots\end{array}\right) \in M_{n}$ is the $n \times n$ matrix of units. Below we omit the index $n$ if it is clear from the context what $n$ is. $E_{i j}$ denotes the tropical matrix which is an analog of the $(i, j)$-th matrix unit, i.e. the matrix with 0 on $(i, j)$-th position and $-\infty$ elsewhere. A matrix with exactly one entry $\alpha$ which is not equal to $-\infty$, i.e., a scalar multiple of a matrix unit is called a cell. For $\alpha \in \mathbb{R}$ we refer to $\alpha \otimes E_{i i}$ as diagonal cells and to $\alpha \otimes E_{i j}$ for $i \neq j$ as off-diagonal cells. We say that $P=\left(p_{i j}\right) \in M_{n}$ is a permutation matrix corresponding to a permutation $\tau \in S_{n}$ if $p_{i \tau(i)}=0$ for all $i=1, \ldots, n$, and all other entries of $P$ are equal to $-\infty$.

Graphs are naturally related to tropical (boolean) matrices: For a square tropical matrix $A=\left(a_{i j}\right)$ of order $n$, its associated digraph is defined to be the digraph $G(A)$ such that $V(G(A))=\left\{v_{1}, \ldots, v_{n}\right\}$ and $G(A)$ has an edge from $v_{i}$ to $v_{j}$ if and only if $a_{i, j} \neq-\infty$. The value $a_{i, j}$ is called weight of the edge from $v_{i}$ to $v_{j}$. Conversely, the adjacency matrix of a digraph $G$ with $V(G)=\left\{v_{1}, \ldots, v_{n}\right\}$ is the square $(-\infty, 0)$-matrix $A(G)$ of order $n$ such that $A(G)(i, j)=0$ if and only if there exists an edge from $v_{i}$ to $v_{j}$ in $G$. If $G$ is a weighted digraph, then its adjacency matrix is usually considered as $A \in M_{n}$, where $a_{i, j}$ is the weight of the edge from $v_{i}$ to $v_{j}$ and $a_{i, j}=-\infty$ if there is no such an edge.

The cyclicity index, or just cyclicity, defined in the next section, is important for determination of regular regimes in scheduling and other network problems, see $[6,10]$ and references therein.

We use a result on matrices in [1] combined with a result on graphs in [11] to give a complete description of the surjective linear transformations of tropical matrices which preserve the cyclicity index.

Our paper is organized as follows. In Section 2 we define the cyclicity index of graphs and matrices and discuss its properties and applications. Section 3 is devoted to the theory of linear maps preserving matrix invariants. We provide a brief historical introduction and several results that we need further. In Section 4 we characterize the bijective linear operators which preserve the cyclicity index. In Section 5 we provide examples of non-bijective transformations preserving the cyclicity index. We leave it open to characterize those.

\section{Cyclicity index of graphs and matrices}

Definition 2.1. The cyclicity index (or just cycliciy) of the digraph $G$ denoted by $\sigma_{G}$ is defined as follows:

(i) If $G$ is strongly connected and $|V(G)| \geq 2$ then its cyclicity equals to the greatest common divisor of the lengths of all dicycles in $G$.

(ii) If $G$ consists of just one vertex with or without loops, then $\sigma_{G}=1$.

(iii) If $G$ is not strongly connected, then its cyclicity equals the least common multiple of the cyclicities of all maximal strongly connected subgraphs of $G$.

To find the cyclicity of a strongly connected component of a digraph one may use the following notion.

Definition 2.2. A digraph $G$ is homomorphic to a digraph $H$ if there exists a surjective map $f: V(G) \rightarrow V(H)$ such that, for any edge $(x, y)$ in $G$ its image $(f(x), f(y))$ is an edge in $H$.

Remark 2.3. The cyclicity of a strongly connected digraph $G$ is the largest $k$ such that $G$ is homomorphic to a dicycle of length $k$. 
Let us think of the elementary dicycle of length $k$ as the digraph whose vertices are the integers modulo $k$, such that $(u, v)$ is an edge if and only if $v=u+1$ modulo $k$. Now to determine if a strongly connected digraph $G$ is homomorphic to the dicycle of length $k$ (defined above) one may proceed by the algorithm:

1. Let $x$ be any vertex of $G$ and put $f(x)=0$ modulo $k$.

2. For any vertex $y$, take a directed path from $x$ to $y$, and let $f(y)$ be the length of that path modulo $k$.

3. Let $V_{i}$ be the set of vertices such that $f(y)=i$.

Now $G$ is homomorphic to the dicycle of length $k$ if and only if any edge $(u, v)$ of $G$ goes from $V_{i}$ to $V_{i+1}$. If this holds then $f$ is a homomorphism from $G$ to the dicycle of length $k$, and the biggest such $k$ is $\sigma(G)$.

Definition 2.4. Let $G=(V, E)$ be a weighted graph with the adjacency matrix $A=\left(a_{i j}\right) \in M_{n}$. Let $C=$ $\left(v_{i_{1}}, \ldots, v_{i_{l}}\right)$ be a dicycle in $G$ with edge weights $a_{i_{1}}, \ldots, a_{i_{l}}$. The average weight of $C$ is the tropical geometric mean of the weights of the edges in $C$ :

$$
w_{a}(C)=\sqrt[\otimes l]{a_{i_{1}} \otimes \ldots \otimes a_{i_{l}}}=\frac{1}{l}\left(a_{i_{1}}+\ldots+a_{i_{l}}\right) .
$$

Note that the tropical geometric mean is precisely the usual arithmetic mean of these values. A dicycle in $G$ is critical if it has the maximal average weight. The critical subgraph $G_{c}$ of $G$ is the union of all critical dicycles in $G$.

Definition 2.5. Let $A \in M_{n}$ be such that its adjacency digraph $G=G(A)$ contains at least one dicycle. Then the cyclicity of $A$ is the cyclicity of the critical graph $G_{c}$ of $G$, i.e., $\sigma(A):=\sigma_{G_{c}}$. If $G(A)$ has no dicycles, then we put $\sigma(A)=1$.

Due to the following fundamental cyclicity theorem [6], cyclicity index is used to investigate periodic behavior of solutions of max-linear systems of type $x(k+1)=A \otimes x(k)$ for $k \geq 0$, where $A$ is an $n \times n$ tropical matrix and $x(0)=x_{0}$ is the initial condition. In particular, it is used for determination of regular regimes in scheduling and other network problems, see [10] and also [6] and references therein.

Theorem 2.6. [6, Theorem 3.9] Let $A$ be an irreducible tropical $n \times n$ matrix with eigenvalue $\lambda$ and cyclicity $\sigma=\sigma(A)$. Then there is a positive integer $N$ such that $A^{\otimes(k+\sigma)}=\lambda^{\otimes \sigma} \otimes A^{\otimes k}$ for all integers $k \geq N$, where $\lambda^{\otimes \sigma}=\underbrace{\lambda \otimes \ldots \otimes \lambda}_{\text {otimes }}$.

\section{Maps preserving matrix invariants}

Given an invariant, a set or a relation on a certain algebraic system, it is natural to ask: what are the transformations of this system that do not change the invariant, set or relation under consideration? In the case of matrix algebras, the investigation of such maps dates back to the result of Frobenius [3], who proved that the bijective linear maps of complex matrices leaving the determinant invariant are precisely those of the form either $X \rightarrow P X Q$ for all $X$ or $X \rightarrow P X^{t} Q$ for all $X$ where $\operatorname{det}(P Q)=1$ and $X^{t}$ denotes the transposed matrix.

This result was subsequently generalized by Schur [9], who gave the characterization of maps preserving all subdeterminants of any fixed order $r$. Later Dieudonné [2] used projective geometry to characterize the bijective linear maps preserving the set of singular matrices over an arbitrary field.

Following these initial investigations, many authors have studied the problem of determining the set of linear operators on the $n \times n$ matrix algebra $M_{n}(F)$ over a field $F$ that leave certain matrix relations, subsets, or properties invariant (see the surveys $[7,8]$ for the details). In the last two decades much attention has been paid to the investigation of maps preserving different invariants for matrices over various semirings.

In the proof of our main result we need the following special case of a result in [1].

Lemma 3.1. [1, Theorem 2.14] Let $T: M_{n} \rightarrow M_{n}$ be a linear transformation (with respect to the tropical operations). Then $T$ is bijective if and only if $T$ is surjective if and only if $T$ induces a bijection on the set of cells. 
The following standard notations are used in the sequel. A linear transformation $T: M_{n} \rightarrow M_{n}$ is called a $(P, B, Q)$-operator, if it has the form $T(X)=P \otimes(X \circ B) \otimes Q$ for all $X \in M_{n}$ or $T(X)=P \otimes(X \circ B)^{t} \otimes Q$ for all $X \in M_{n}$, where $\circ$ denotes the Schur (Hadamard), i.e. element-wise product: $(X \circ B)_{i j}=x_{i j} \otimes b_{i j}$, and $P, Q$ are tropical products of permutation matrices and invertible diagonal matrices, and $B$ has no $-\infty$ entries. $T$ is usually called standard if it is a $(P, B, Q)$-operator, where $B=J$. The term standard reflects that most of the linear preservers over semirings lie in this class, see for example, $[1,4,5,8]$.

\section{Characterization of surjective linear maps preserving cyclicity}

In this section we investigate linear transformations on matrices which preserve the cyclicity index. We shall use a result in [11] on edge-bijections induced by isomorphisms or anti-isomorphisms. An isomorphism (respectively anti-isomorphism) of a digraph $D$ onto a digraph $D^{\prime}$ is a bijection of the vertex set $V(D)$ onto the vertex set $V\left(D^{\prime}\right)$ such that, for any vertices $x, y \in V(D)$, there is a directed edge from $x$ to $y$ if and only if there is a directed edge from $f(x)$ to $f(y)$ (respectively from $f(y)$ to $f(x)$ ). In either case, $f$ induces an edge-bijection $\pi$ of $E(D)$ onto $E\left(D^{\prime}\right)$ such that $\pi$ and $\pi^{-1}$ preserve dicycles. (Recall that undirected graph is called connected if for each pair of its vertices there is a non-oriented path between them, and it is called $k$-connected if it has more than $k$ vertices and remains connected whenever less than $k$ vertices are removed.) Conversely, if the underlying graph of $D$ is 3-connected, then any edge-bijection $\pi$ of $E(D)$ onto $E\left(D^{\prime}\right)$ with the property that $\pi$ and $\pi^{-1}$ preserve dicycles is induced by an isomorphism or anti-isomorphism, as proved in [11, Theorem 3.3]. (Note that, if the underlying graph of $D$ is 3-connected, then there are no so-called 2-switches in [11, Theorem 3.3]).

Theorem 4.1. Let $T: M_{n} \rightarrow M_{n}$ be a surjective linear transformation, which preserves the cyclicity. Then there exists a matrix $B \in M_{n}$, a permutation matrix $P \in M_{n}$, and a permutation $\rho \in S_{n}$ such that $T$ is a composition of some of the following transformations:

1) $X \rightarrow P \otimes X \otimes P^{t}$ for all $X \in M_{n}$,

2) $X \rightarrow X \circ B$ for all $X \in M_{n}$, where $B$ has no $-\infty$ entry, and $\circ$ is defined at the end of the previous section.

3) $X=\left(x_{i j}\right) \rightarrow Y=\left(y_{i j}\right)$, where $y_{i j}=x_{i j}$ for $i \neq j$ and $y_{i i}=x_{\rho(i) \rho(i)}$ for all $i=1, \ldots, n$.

4) $X \rightarrow X^{t}$ for all $X \in M_{n}$.

Proof. 1. By Lemma 3.1 the map $T$ permutes the cells. By assumption, $T$ is linear. Then $T$ gives rise to a permutation, which we call $\tau$, of the directed edges in the complete digraph with $n$ vertices. Namely, let $e$ be the edge from $i$ to $j$. Then $\tau(e)$ is the edge from $k$ to $l$, where $k$ and $l$ are defined by $T\left(E_{i j}\right)=\alpha \otimes E_{k l}$.

2. If $L$ is a set of directed edges, then we let $A(L)$ denote the matrix which is the tropical sum of all matrices $E_{i j}$ such that the edge $(i, j)$ is in $L$.

3. Let us first prove that if $C$ is a dicycle of length $>1$ in the complete digraph, then $\tau(C)$ is also a dicycle.

Indeed, the cyclicity index of $A(C)$ is the length of $C$, thus $\sigma(A(C))>1$. Since $T$ preserves the cyclicity index, $\sigma(T(A(C)))>1$. Hence, the adjancy digraph of $T(A(C))$ contains a dicycle $D$. This dicycle $D$ must be equal to the whole graph of $T(A(C))$. For otherwise, since $T$ is a bijection on the set of cells, there is a proper edge set of $C$, say $C^{\prime}$, such that $\tau\left(C^{\prime}\right)=D$. However $\sigma_{C^{\prime}}=1$ and $\sigma_{D}>1$, which is a contradiction. If $C$ is a dicycle of length 1 in the complete digraph, then clearly $\tau(C)$ is also a dicycle of length 1 because $T$ permutes the cells.

4. It follows from Item 3 that a set $C$ of edges in the complete digraph forms a dicycle if and only if $\tau(C)$ forms a dicycle.

5. In [11, Theorem 3.3] it is proved that then the restriction of $\tau$ to the non-diagonal entries is induced by an isomorphism or an anti-isomorphism of the complete digraph onto itself. In order to apply [11, Theorem 3.3] we put $D=F$ in the complete digraph, and we let $\pi=\tau$. 
6. By Item 5 there is a permutation $\delta$ of indices $\{1,2, \ldots, n\}$ such that $T\left(E_{i j}\right)=b_{i j} \otimes E_{\delta(i) \delta(j)}$ for all $1 \leq i \neq$ $j \leq n$. Let $P$ be the permutation matrix corresponding to $\delta$. By the last remark in Item 3 , there is a permutation $\rho$ of the indices $\{1,2, \ldots, n\}$ such that $T\left(E_{i i}\right)=b_{i i} \otimes E_{\rho(i) \rho(i)}$ for all $1 \leq i \leq n$.

This proves Theorem 4.1.

We shall now investigate to which extent the converse of Theorem 4.1 holds.

Lemma 4.2. Let $T: M_{n} \rightarrow M_{n}$ and $P \in M_{n}$ be a permutation matrix.

If $T$ satisfies 1) or 4) in Theorem 4.1, then $T$ preserves the cyclicity.

Proof. 1) Multiplication with $P$ on the left and $P^{t}$ on the right changes the enumeration of vertices, i.e., it corresponds to an automorphism of the graph. Hence, it does not change the critical subgraph and does not change cyclicity.

2) Transposition reverses the directions of all edges. Thus it reverses the directions of all dicycles, i.e., it does not change their lengths. In other words, transposition of the graph matrix corresponds to an antiisomorphism in the adjacency digraph. Hence, it does not change the critical subgraph and does not change cyclicity.

Lemma 4.3. Let $T: M_{n} \rightarrow M_{n}$. Assume that there exists a matrix $B$ without $-\infty$ entry and a permutation $\rho \in S_{n}$ such that $T$ satisfies 2) or 3) in Theorem 4.1. Then T preserves the cyclicity index if and only if $\rho=\delta$ and $B=\alpha \otimes J$ for some $\alpha \in \mathbb{R}$, i.e., $B$ is the matrix with all entries equal to $\alpha$.

Proof. The "if"-part is trivial. We now prove the "only if"-part. Let $\delta$ and $\rho$ be as in Item 5 and Item 6 in the proof of Theorem 4.1. By Lemma 4.2 without loss of generality we may assume that $\delta$ is the identical permutation. Consider first the case where $\rho=\delta=i d$. Let $\alpha \in \mathbb{R}$ be the maximum entry of $B$. Suppose (reductio ad absurdum) that Lemma 4.3 is false. Then there exist distinct indices $i, j$ such that at least one of $b_{i, i}, b_{i, j}, b_{j, i}$ equals $\alpha$, and at least one of $b_{i, i}, b_{i, j}, b_{j, i}$ is strictly less than $\alpha$. Without loss of generality we assume that $i=1, j=2$. We consider the case where $b_{1,1}=b_{1,2}>b_{2,1}$. The other cases are similar. Let $X_{0}=\left(x_{i j}\right)$ be a matrix such that each of $x_{1,1}, x_{1,2}, x_{2,1}$ is distinct from $-\infty$, and all other $x_{i, j}$ are equal to $-\infty$ and such that $x_{1,1}+b_{1,1}=\left(x_{1,2}+b_{1,2}+x_{2,1}+b_{2,1}\right) / 2$. Then $x_{1,1}<\left(x_{1,2}+x_{2,1}\right) / 2$ and hence the critical subgraph of $G\left(X_{0}\right)$ is a dicycle of length 2, which implies that $X_{0}$ has cyclicity index 2 . However, $T\left(X_{0}\right)$ has cyclicity index 1, because the critical subgraph of $G\left(T\left(X_{0}\right)\right)$ consists of a dicycle of length 1 and a dicycle of length 2 having a vertex in common, a contradiction.

Consider next the case where $\rho \neq i d$. We consider here the case where $n \geq 4$ and leave the case where $n<4$ to the reader. Since $\rho \neq i d$ and $n \geq 4$, there are indices $i, j$ such that $i, j, k, m$ are all distinct where $k=\rho(i), m=\rho^{-1}(i)$. We also choose $i$ such that $b_{i, i}$ is minimum among all $b_{\rho^{q}(i), \rho^{q}(i)}$, where $q$ is an integer. If $b_{i, i} \leq\left(b_{i, j}+b_{j, i}\right) / 2$, then we define $X_{0}$ as in the first case and obtain a contradiction as in the the first case. If $b_{i, i} \geq\left(b_{i, j}+b_{j, i}\right) / 2$, then we let $X_{1}=\left(x_{i j}\right)$ be a matrix such that each of $x_{m, m}, x_{i, j}, x_{j, i}$ is distinct from $-\infty$, and all other entries are equal to $-\infty$ and such that $x_{m, m}+b_{m, m}=\left(x_{i, j}+b_{i, j}+x_{j, i}+b_{j, i}\right) / 2$. Then $T\left(X_{1}\right)$ has cyclicity index 1 , because the critical subgraph of $G\left(T\left(X_{1}\right)\right)$ consists of a dicycle of length 1 and a dicycle of length 2 having a vertex in common. However, $X_{1}$ has cyclicity index 2 because the critical subgraph of $G\left(X_{1}\right)$ consists of a dicycle of length 2 . This contradiction completes the proof.

Theorem 4.4. Let $T: M_{n} \rightarrow M_{n}$ be a surjective linear map. Then $T$ preserves cyclicity if and only if there exist a permutation matrix $P \in M_{n}$ and an $\alpha \in \mathbb{R}$, such that $T$ has the form $T(X)=\alpha \otimes P \otimes X \otimes P^{t}$ for all $X \in M_{n}$, or $T(X)=\alpha \otimes P \otimes X^{t} \otimes P^{t}$ for all $X \in M_{n}$.

Proof. The "only if" part holds by Theorem 4.1 and Lemma 4.3. The "if" part follows from Lemma 4.2.

We say that $T$ preserves cyclicity index 1 if, for each matrix $A, \sigma(A)=1$ if and only if $\sigma(T(A))=1$. A close inspection of the proof of Lemma 4.3 above also implies the following. 
Corollary 4.5. Let $T: M_{n} \rightarrow M_{n}$ be a surjective linear map. Then $T$ preserves cyclicity index 1 if and only if there exist a permutation matrix $P \in M_{n}$ and an $\alpha \in \mathbb{R}$, such that $T$ has the form $T(X)=\alpha \otimes P \otimes X \otimes P^{t}$ for all $X \in M_{n}$, or $T(X)=\alpha \otimes P \otimes X^{t} \otimes P^{t}$ for all $X \in M_{n}$.

\section{Non-surjective linear maps preserving cyclicity}

Proposition 5.1. There exist non-surjective linear maps $\varphi: M_{2} \rightarrow M_{2}$ that preserve the cyclicity index.

Proof. Define

$$
\varphi\left(\begin{array}{ll}
a_{11} & a_{12} \\
a_{21} & a_{22}
\end{array}\right)=\left(\begin{array}{cc}
-\infty & a_{12} \\
a_{21} & a_{11} \oplus a_{22}
\end{array}\right)=\left(a_{i j}^{\prime}\right)
$$

for all $A=\left(a_{i j}\right) \in M_{2}$.

Clearly, $\varphi$ is not surjective. We now prove that $\varphi$ preserves cyclicity.

Any digraph with 2 vertices

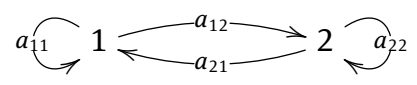

has cyclicity index is either 1 or 2 . Hence, the cyclicity index of a $2 \times 2$ matrix is either 1 or 2 . By the definition

of $\varphi, G(\phi(A))$ is

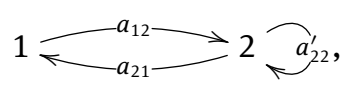

where $a_{22}^{\prime}=a_{11} \oplus a_{22}=\max \left(a_{11}, a_{22}\right)$.

Consider first the case $\sigma(A)=1$. If $G(A)$ has a dicycle of length 2, then its average weight is less than or equal to the maximum of the weights of the loops, that is, $\max \left(a_{11}, a_{22}\right) \geq \frac{a_{12}+a_{21}}{2}$. Hence

$$
\max \left(a_{11}^{\prime}, a_{22}^{\prime}\right)=\max \left(-\infty, \max \left(a_{11}, a_{22}\right)\right) \geq \frac{a_{12}^{\prime}+a_{21}^{\prime}}{2},
$$

and so, the critical subgraph contains a loop which implies $\sigma(\varphi(A))=1$. If $G(A)$ has no dicycle of length 2, then $a_{12}$ or $a_{21}$ is $-\infty$ and hence either $a_{12}^{\prime}$ or $a_{21}^{\prime}$ is $-\infty$, so, there is no dicycle of length 2 in $G\left(A^{\prime}\right)$ either. Again we conclude that $\sigma(\varphi(A))=1$.

Consider next the case $\sigma(A)=2$. Then $\frac{a_{12}+a_{21}}{2}>\max \left(a_{11}, a_{22}\right)$. By the definition of $\varphi$ we have

$$
\max \left(a_{11}^{\prime}, a_{22}^{\prime}\right)=\max \left(-\infty, \max \left(a_{11}, a_{22}\right)\right)<\frac{a_{12}^{\prime}+a_{21}^{\prime}}{2},
$$

and $\sigma(\varphi(A))=2$.

Hence $\varphi$ preserves cyclicity.

Acknowledgments: The authors are grateful to the referee for useful suggestions.

The research of the first and the second authors is financially supported by RSF Project N. 17-11-01124. The third author is supported by Independent Research Fund Denmark, 8021-002498 AlgoGraph.

Data Availability Statement: Data sharing is not applicable to this article as no datasets were generated or analysed during the current study.

\section{References}

[1] L. Beasley, A. Guterman, Linear preservers of extremes of rank inequalities over semirings: the factor rank, J. of Math. Sciences. 2005, 131(5), 5919-5938.

[2] J. Dieudonné, Sur une généralisation du groupeorthogonal à quatre variables, Arch. Math. 1949, 1, 282-287. 
[3] G. Frobenius, Über die Darstellung der endlichen Gruppen durch lineare Substitutionen, Sitzungsber, Preuss. Akad. Wiss (Berlin), Berlin, 1897, pp. 994-1015.

[4] A. Guterman, Matrix invariants over semirings, Handbook of Algebra ed.: M. Hazewinkel, Elseiver, North-Holland, 2009, 6, 3-33.

[5] A. Guterman, M. Johnson, M. Kambites, Linear isomorphisms preserving Green's relations for matrices over anti-negative semifields, Linear Algebra Appl. 2018, 545, 1-14.

[6] B. Heidergott, G.J. Olsder, J.van der Woude, Max Plus at Work, Princeton: Princeton University Press, 2006.

[7] C.-K. Li and N.K. Tsing, Linear preserver problems: A brief introduction and some special techniques, Linear Algebra Appl. 1992, 162-164, 217-235.

[8] S. Pierce and others, A survey of linear preserver problems, Linear and Multilinear Algebra, 1992, 33, 1-119.

[9] I. Schur, Einige Bemerkungen zur Determinantentheorie. Akad. Wiss. Berlin: S.-Ber. Preuß., 1925 454-463.

[10] S. Sergeev, Max algebraic powers of irreducible matrices in theperiodic regime: An application of cyclic classes, Linear Algebra Appl., 2009, 431, 1325-1339.

[11] C. Thomassen, Whitney's 2-switching theorem, cycle spaces, and arc mappings of directed graphs, J. Combinatorial Theory, Ser. B, 1989, 46, 257-291. 